\title{
ANÁLISE DO CONHECIMENTO DE PAIS OU RESPONSÁVEIS SOBRE A SAÚDE BUCAL DOS FILHOS COM NECESSIDADES ESPECIAIS
}

\author{
Maria Cláudia Celestino CRESCÊNCIO' \\ mariaclaudiacrescencio@gmail.com \\ Débora Passos CRISTIANO² \\ debycristiano@hotmail.com \\ Priscyla Waleska SIMÕES 3 \\ pri@unesc.net \\ Fernanda Guglielmi Faustini SONEGO ${ }^{4}$ \\ fgfsonego@unesc.net
}

\section{RESUMO}

Introdução: Devido ao comprometimento físico ou mental, os pacientes com necessidades especiais apresentam dificuldades em realizar sua higiene bucal e assim muitos deles acabam recebendo auxílio de outra pessoa, seja um familiar ou cuidador. Objetivo: Buscou-se avaliar o conhecimento de pais ou responsáveis, sobre a saúde bucal dos seus filhos com necessidades especiais, em uma Clínica Odontológica Universitária Catarinense. Métodos: Foi realizado um estudo transversal, descritivo, com abordagem quantitativa. A população foi composta por pais ou responsáveis pelos pacientes que frequentaram uma Clínica Odontológica Universitária no município de Criciúma/SC, de agosto a setembro de 2016. Dos questionários aplicados, foram excluídos os que não estavam respondidos corretamente, resultando no total de 32 questionários. Resultados: Quanto à participação da higiene bucal dos pacientes, 51,6\% auxiliam seus filhos. Observou-se que a prática da escovação dental ocorre de duas a três vezes ao dia em grande parte dos pacientes $(71,9 \% ; n=23)$, com relevância estatística $(p<0,007)$. O esclarecimento sobre saúde bucal aos filhos é dado pelos pais ou responsáveis $(78,1 \%)$, sendo mais frequentemente instruídos pela mãe $(75,0 \%$; $\mathrm{n}=18$ ). Metade dos pais/responsáveis classificou a saúde bucal do seu filho como uma condição oral boa $(50,0 \%)$. Entre os aspectos facilitadores relatados pelos responsáveis para manter a saúde bucal do $\mathrm{PNE}$, 38,7\% sugeriram a instalação de centros de referência habilitados e maior número de profissionais, além do trabalho multidisciplinar (19,4\%). Conclusão: Os pais/responsáveis dos pacientes com necessidades especiais apresentaram conhecimento satisfatório sobre a saúde bucal de seus filhos.

DESCRITORES: SAÚDE BUCAL. PESSOAS COM DEFICIÊNCIA. CONHECIMENTO.

1 Graduanda em Odontologia - Universidade do Extremo Sul Catarinense

2 Cirurgiã-Dentista residente do Programa de Residência Multiprofissional em Atenção Básica/Saúde da Família - Universidade do Extremo Sul Catarinense.

3 Doutora em Ciências da Saúde - Universidade do Extremo Sul Catarinense. Professora do Curso de Odontologia e Professora Pesquisadora no Programa de Pós-Graduação em Saúde Coletiva (PPGSCol) - Universidade do Extremo Sul Catarinense.

4 Mestre em Odontopediatria pelo C.P.O/São Leopoldo Mandic. Professora de Odontopediatria e Saúde Coletiva no Curso de Odontologia - Universidade do Extremo Sul Catarinense. 


\section{ANALIYSIS OF KNOWLEDGE OF PARENTS OR GUARDIANS ABOUT THE ORAL HEALTH OF THEIR CHILDREN WITH SPECIAL NEEDS}

\section{ABSTRACT}

Introduction: Due to the physical or mental impairment, patients with special needs have restrictions when performing their oral hygiene, so many of them end up getting help from another person, whether a relative or caregiver. Objective: We sought to evaluate the knowledge of parents or guardians on the oral health of their children with special needs in a University Dental Clinic in Santa Catarina. Methods: A transverse study, descriptive with quantitative approach was held. The population was composed by parents or responsibles by patients that attended the Dental Clinic at the University in the town of Criciúma/ SC between August 2016 till September 2016. From the questionnaires applied were excluded the ones that were not answered correctly, resulting in the total of 32 questionnaires. Results: Regarding the oral hygiene of the patients, $51,6 \%$ help their children. Was observed that the practice of the dental brushing occurs twice to three times a day, a big part of the patients $(71,9 \% ; n=23)$, with relevant statistics $(p<0,007)$ the clarification about oral health to the children is given by parents or responsible $(78,1 \%)$, being more frequently instructed by the mother $(75,0 \% ; n=18)$. Half of the parents/ responsible rated the oral health condition of their children as good (50,0\%). Between the aspects reported by the responsible for keeping the oral health of the patients with special needs, 38,7\% claim on installing qualified referral centers and more professionals; beyond to the multidisciplinary work (19.4\%). Conclusions: Parents and/or guardians of patients with special needs have satisfactory knowledge about the oral health of their children.

DESCRIPTORS: ORAL HEALTH. DISABLED PERSONS. KNOWLEDGE.

\section{INTRODUÇÃO}

Pacientes com necessidades especiais (PNE) apresentam um comprometimento físico e/ou mental que, em alguns casos, torna a realização de algumas atividades diárias difíceis de serem efetivadas e assim muitos deles acabam recebendo auxílio de outra pessoa, de um familiar ou cuidador'.

No Brasil, a deficiência é classificada pelo grau de severidade de acordo com a percepção do indivíduo, ou seja, nesse contexto é considerada deficiência até mesmo uma pequena alteração visual. Segundo o Instituto Brasileiro de Geografia e Estatística (IBGE), no ano de 2000, cerca de 14,5\% da população brasileira apresentou alguma deficiência, e em 2010 esse percentual aumentou para 23,9\% da população brasileira, correspondendo a cerca de 45 milhões de brasileiros declarados com deficiência ${ }^{2,3}$.

Parte da população com necessidade especial possui a saúde bucal comprometida, pela presença da doença cárie e/ou periodontal. Verifica-se que somente $3 \%$ da população com deficiência têm acesso ao tratamento odontológico ${ }^{4}$. Esse fato pode estar relacionado à condição socioeconômica, ao desconhecimento, à não colaboração do paciente, desmotivação dos cuidadores e despreparo do dentista no atendimento ${ }^{5,6,7}$.

Atualmente, a Odontologia tem evidenciado uma mudança no processo saúde-doença, embasada nas políticas públicas que atendam às necessidades específicas da população e na humanização do cuidado ${ }^{8}$.

O cirurgião-dentista precisa conhecer os mais variados tipos de deficiência e seus efeitos deletérios à saúde bucal do indivíduo. Énecessário utilizar uma abordagem multidisciplinar, respeitando a particularidade 
ISSN 1983-5183

de cada paciente e, para que haja efetividade em relação às mudanças de hábitos do paciente, é importante a participação dos pais ou cuidadores, caso contrário, o processo de educação pode não alcançar êxito ${ }^{3}$.

Assim, o desconhecimento sobre cuidados necessários de higiene bucal representa um fator a ser considerado, uma vez que a informação, embora disponível, não chega a toda a população de igual forma, e dificilmente é apreendida de modo que produza conhecimento e autonomia em relação aos cuidados com a saúde?.

De acordo com o que foi supracitado, objetivou-se avaliar o conhecimento de pais ou responsáveis, sobre a saúde bucal dos seus filhos com necessidades especiais, em uma Clínica Odontológica Universitária Catarinense.

\section{MÉTODOS}

Trata-se de um estudo transversal, quantitativo, de análise descritiva, cuja coleta de dados ocorreu em uma Clínica Odontológica Universitária (COU), no período de agosto a setembro 2016. 0 projeto de pesquisa foi aprovado pelo Comitê de Ética da Universidade do Extremo Sul Catarinense (UNESC), sob parecer no 1.644.489/2016.

Foram incluídos no estudo 32 pais ou responsáveis, juntamente com seus filhos portadores de necessidades especiais, que frequentaram a COU, mediante a assinatura do Termo de Consentimento Livre e Esclarecido, seguindo a resolução n 466/12 do Conselho Nacional de Saúde. Foram excluídos os acompanhantes menores de idade e os que não possuíam uma ligação direta e diária com os PNE.

Foi aplicado aos pais ou responsáveis um questionário semiestruturado, adaptado dos estudos de Marra $^{10}$ (2007)e Pereira"1 (2010), contendo 43 perguntas relacionadas ao conhecimento sobre a saúde bucal deles e de seus filhos, visitas ao dentista, acompanhamento médico, indicação do pediatra ao dentista, comportamento do PNE nas consultas odontológicas, condição de saúde bucal do paciente e pais, bem como sobre a condição socioeconômica da família.

Na sequência, foi realizada a análise dos prontuários dos pacientes com necessidades, cujos pais ou responsáveis responderam ao questionário. Nesta análise, verificou-se a presença ou não de doença cárie e ou periodontal. Para realização do estudo, foram seguidas as normas de ingresso na COU, utilizando-se equipamentos de proteção individual, como luvas, máscara, touca, óculos e jaleco.

Após a coleta de dados, foi elaborado, em planilhas do software Microsoft Excel, um banco, para uma melhor organização e apresentação dos dados.

Na sequência, o banco de dados foi exportado para o software Statistical Package for the Social Sciencies (SPSS) 22.0, aplicativo também utilizado para a análise estatística. Foram também calculadas algumas medidas descritivas como média, desvio-padrão, mediana e intervalo interquartil, considerandose a distribuição não gaussiana para as variáveis quantitativas e frequência absoluta e relativa para as qualitativas.

O teste de Qui-quadrado de Pearson foi utilizado para quantificar a associação ou independência entre as variáveis categóricas e escolaridade. Para as associações estatisticamente significativas foi realizada a análise de resíduos ajustados, buscando-se identificar onde estavam tais associações. Para todos os testes supracitados foi considerado como significativo $a=0,05$ e confiança de $95 \%$. 
ISSN 1983-5183

\section{RESULTADOS}

Participaram do estudo 32 pais ou responsáveis, cujos filhos são portadores de necessidades especiais, que frequentaram a Clínica Odontológica Universitária, na disciplina de Odontologia para PNE, no período de agosto a setembro de 2016.

Dos pais ou responsáveis $71,8 \%$ ( $n=23)$ eram do gênero feminino e $25,0 \%(n=8)$ do masculino. Quanto ao gênero dos filhos participantes, $54,8 \%$ ( $n=17)$ eram do gênero feminino e $45,2 \%(n=14)$ do masculino.

Quando analisado o diagnóstico dos PNE, constatou-se por intermédio dos entrevistados que $25,0 \%$ $(n=8)$ deles apresentam Síndrome de Down, 15,6\% $(n=5)$ deficiência mental e 9,4\% $(n=3)$ paralisia cerebral.

Na Tabela 1 estão as respostas dos pais ou responsáveis acerca do conhecimento deles sobre a saúde bucal de seus filhos, como também abordagens socioeconômicas.

Tabela 1 - Perfil e conhecimento dos pais sobre a saúde bucal de seus filhos PNE.

\begin{tabular}{lc}
\hline \multicolumn{1}{c}{ Variáveis } & $\mathrm{n}(\%) \mathrm{n}=32$ \\
\hline Orientação do pediatra sobre higiene bucal & $18(56,3)$ \\
Sim & $14(43,8)$ \\
Não & \\
O pediatra indicou que procurasse um dentista & $15(46,9)$ \\
Sim & $17(53,1)$ \\
Não & \\
Preocupação do médico com saúde bucal do seu filho & \\
Sim & $13(41,9)$ \\
Não & $18(58,1)$ \\
Seu filho já foi ao dentista? & $32(100,0)$ \\
Sim & \\
Precisou de tratamento dentário & $29(90,6)$ \\
Sim & $3(9,4)$ \\
Não & \\
Por que levou seu filho(a) ao dentista (na primeira vez) & $21(65,6)$ \\
Consulta de rotina & $9(28,1)$ \\
Emergência (dor) & \\
Você (ou algum adulto) ajuda na escovação do filho & $16(51,6)$ \\
Sim & $15(48,4)$ \\
Não & \\
Quantas vezes por dia seu filho(a) escova os dentes & $5(15,6)$ \\
Duas a três vezes ao dia & $23(71,9)$ \\
\hline
\end{tabular}


ISSN 1983-5183

\begin{tabular}{|c|c|}
\hline Variáveis & $n(\%) n=32$ \\
\hline Somente uma vez ao dia & $4(12,5)$ \\
\hline \multicolumn{2}{|l|}{ Ele (a) faz bochecho com flúor } \\
\hline Sim & $10(31,3)$ \\
\hline Não & $22(68,8)$ \\
\hline \multicolumn{2}{|l|}{ Teve sangramento gengival } \\
\hline Sim & $11(34,4)$ \\
\hline Não & $21(65,6)$ \\
\hline \multicolumn{2}{|l|}{ Dentista indicou sedação ou anestesia geral } \\
\hline Sim & $10(31,3)$ \\
\hline Não & $22(68,8)$ \\
\hline \multicolumn{2}{|l|}{ Quem ensinou seu filho(a) sobre saúde bucal } \\
\hline Mãe ou pai & $25(78,1)$ \\
\hline Dentista & $7(21,9)$ \\
\hline \multicolumn{2}{|l|}{ Como classificaria a saúde bucal do seu filho } \\
\hline Ruim & $3(9,4)$ \\
\hline Regular & $13(40,6)$ \\
\hline Boa & $16(50,0)$ \\
\hline \multicolumn{2}{|l|}{ Qual o salário médio da família } \\
\hline 2 salários mínimos & $16(51,6)$ \\
\hline 3 salários mínimos & $8(25,8)$ \\
\hline \multicolumn{2}{|l|}{ Tem plano de saúde } \\
\hline Sim & $7(21,9)$ \\
\hline Não & $25(78,1)$ \\
\hline \multicolumn{2}{|l|}{ Ao adoecer, onde procura atendimento } \\
\hline SUS & $27(84,4)$ \\
\hline Consultório particular & $2(6,3)$ \\
\hline Mediana dos meses de indicação ao dentista (II) & $1,00(1,00-4,25)$ \\
\hline
\end{tabular}

(II) Intervalo interquartil

Observou-se que $56,3 \%$ ( $n=18$ ) receberam orientações, quanto à higiene oral de seu filho, do médico pediatra. Ao correlacionar os dados entre a orientação do pediatra quanto à higiene bucal e o gênero, não houve diferença significativa $(p=0,681)$. Avaliando o encaminhamento do PNE ao dentista, pelo pediatra, verificou-se que $53,1 \%(n=17)$ deles não foram referenciados. A mediana de idade dos pacientes PNE indicados para o dentista foi de 1,00 (1,00-4,25) meses.

Quando analisada a preocupação do médico pediatra com a saúde bucal do seu filho, pouco mais da metade alegou desinteresse do profissional $(58,1 \% ; n=18)$. Na associação faixa etária, da primeira visita do 
ISSN 1983-5183

PNE ao dentista e a saúde bucal do mesmo, a mediana encontrada foi de 5,00 (2,00-8,00) anos.

Os pais foram unânimes em relatar sobre a ida de seus filhos ao dentista (100,0\%; $n=32)$. Dentre estes, $90,6 \%$ ( $n=29)$ necessitaram de tratamento dentário, sendo o PNE do gênero feminino a maior prevalência $(91,7 \% ; n=22)$.

O motivo que levou os pais a procurarem a assistência odontológica pela primeira vez foi, em grande parte, para uma consulta de rotina $(65,6 \% ; n=21)$. Em se tratando do comportamento do PNE no dentista, $43,8 \%(n=14)$ relataram que permaneceram calmos, $21,9 \%(n=7)$ que choraram durante a consulta e $18,6 \%$ $(n=6)$ deles não deixaram tratar. Observou-se que 68,8\% (n=22) não têm medo de dentista.

Na análise quanto ao esclarecimento do dentista sobre o problema de seu filho, 76,7\% ( $n=23$ ) declararam que houve tal explicação.

Quando perguntados em qual local foi realizado o tratamento dentário, 43,8\% ( $n=14)$ responderam consultório particular e hospital público; sendo que 34,4\% (n=11) foram atendidos em instituições e hospital público.

Verificou-se que os PNE que frequentaram a COU não exibiram comprometimento motor ou doença crônica que comprometa a realização da higiene bucal $(78,1 \% ; n=25)$

Quando indagados sobre sua participação na higiene bucal dos filhos, os pais ou responsáveis responderam que auxiliam seus filhos no desempenho dessa função $(51,6 \%, n=16)$. Observou-se que a prática da escovação dental ocorre de duas a três vezes ao dia, em grande parte dos pacientes (71,9\%; $\mathrm{n}=23$ ). Esses resultados mostraram correlação estatisticamente significativa entre o gênero dos pais e a quantidade de escovação dental realizada, por dia, pelos pacientes $(p=0,007)$. Verificou-se que $68,8 \%(n=22)$ dos filhos não realizam bochecho com flúor. Ao fazer higiene oral de seu filho, os pais relataram não observar sangramento gengival em $65,6 \%(n=21)$ deles.

A maioria dos pais ou responsáveis revelou não ter sido orientado quanto à possibilidade de tratamento dentário com sedação ou anestesia geral, correspondendo a 68,8\% ( $n=22)$. Embora os resultados possam sugerir associação entre sexo e o repasse dessa informação ao responsável do PNE, não houve diferença significativa $(p=0,709)$.

A Tabela 1 descreve que quem ensinou o filho sobre saúde bucal foram os próprios pais $(78,1 \% ; n=25)$, sendo mais frequentemente a mãe $(75,0 \% ; n=18)$.

Metade dos participantes classificou a condição bucal do seu filho como boa $(50,0 \% ; n=16)$. Os que referiram ser regular foram $40,6 \%(n=13)$.

Para facilitar a manutenção da saúde bucal do PNE, os pais relataram sobre a instalação de centros de referência habilitados e maior números de profissionais (38,7\%; $n=12)$, além do trabalho multidisciplinar $(19,4 \% ; n=6)$.

Quando os participantes foram questionados sobre como avaliam sua própria saúde bucal, 43,8\% $(n=14)$ a consideraram boa. Classificaram como ruim 31,3\% $(n=10)$ e $21,9 \%(n=7)$ regular. Resultados sem significância estatística $(p=0,487)$.

Por meio das informações obtidas junto aos entrevistados sobre a sua escolaridade, verificou-se 
ISSN 1983-5183

que $67,7 \%$ ( $n=21$ ) possuem ensino médio ou superior; e os que nunca foram ao colégio ou têm ensino fundamental foram $32,3 \%(n=10)$.

A renda mensal do chefe de família ficou assim expressa: recebem dois salários mínimos 51,6\% ( $n=16)$, três salários mínimos 25,8\% ( $n=8)$, de quatro a dez salários mínimos 12,9\% ( $n=4)$ e 1 salário mínimo 9,7\% $(n=3)$.

Os resultados obtidos sobre a moradia dos participantes demonstraram que $78,1 \%(n=25)$ deles têm casa própria e $81,3 \%(n=26)$ moram em casa de alvenaria.

Quando questionados sobre plano de saúde, verificou-se que 21,9\% ( $n=7)$ possuem e a maioria, quando adoece, procura atendimento no Sistema Único de Saúde $(84,4 \%$; $=27)$.

Nas análises dos prontuários odontológicos, observou-se que $68,8 \%(n=22)$ dos pacientes PNE possuem atividade de cárie e, no que diz respeito à doença periodontal, constatou-se que $75,0 \%$ ( $n=24)$ não apresentam essa doença.

Tabela 2 - Análise estratificada por escolaridade dos pais e/ou responsáveis.

\begin{tabular}{|c|c|c|c|c|c|c|}
\hline \multirow[b]{2}{*}{ Variáveis } & \multicolumn{4}{|c|}{ Escolaridade } & \multirow[b]{2}{*}{$\begin{array}{l}\text { Médio } \\
\text { Completo } \\
\mathrm{n}=10 \mathrm{n}(\%)\end{array}$} & \multirow[b]{2}{*}{ Valor $p$} \\
\hline & $\begin{array}{c}\text { Total } \\
\mathrm{n}=32 \mathrm{n}(\%)\end{array}$ & $\begin{array}{c}\text { Nunca } \\
\text { estudou } \\
\mathrm{n}=5 \mathrm{n}(\%)\end{array}$ & $\begin{array}{c}\text { Fund. } \\
\text { Completo } \\
\mathrm{n}=5 \mathrm{n}(\%)\end{array}$ & $\begin{array}{c}\text { Médio } \\
\text { Incompleto } \\
\mathrm{n}=5 \mathrm{n}(\%)\end{array}$ & & \\
\hline \multicolumn{7}{|c|}{ Saúde bucal dos filhos } \\
\hline Ruim & $3(9,7)$ & & $2(40,0)$ & $1(20,0)$ & & 0,160 \\
\hline Regular & $12(38,7)$ & $2(40,0)$ & $1(20,0)$ & $3(60,0)$ & $5(50,0)$ & \\
\hline Boa & $16(51,6)$ & $3(60,0)$ & $2(40,0)$ & $1(20,0)$ & $5(50,0)$ & \\
\hline \multicolumn{7}{|l|}{ Filho com cárie } \\
\hline Sim & $21(67,7)$ & $5(100,0)$ & $4(80,0)$ & $3(60,0)$ & $5(50,0)$ & 0,366 \\
\hline Não & $10(32,3)$ & & $1(20,0)$ & $2(40,0)$ & $5(50,0)$ & \\
\hline \multicolumn{7}{|l|}{$\begin{array}{l}\text { Filho com doença } \\
\text { periodontal }\end{array}$} \\
\hline Sim & $8(25,8)$ & $2(40,0)$ & $1(20,0)$ & & $4(40,0)$ & 0,453 \\
\hline Não & $23(74,2)$ & $3(60,0)$ & $4(80,0)$ & $5(100,0)$ & $6(60,0)$ & \\
\hline \multicolumn{7}{|c|}{ Quem ensinou seu filho sobre Saúde Bucal? } \\
\hline Mãe ou pai & $25(80,6)$ & $3(60,0)$ & $5(100,0)$ & $2(40,0)$ & $9(90,0)$ & 0,043 \\
\hline Dentista & $6(19,4)$ & $2(40,0)$ & & $3(60,0)$ & $1(10,0)$ & \\
\hline
\end{tabular}

Na Tabela 2, observou-se que, mesmo sendo elevado o grau de escolaridade dos pais, quando o mesmo foi associado à presença de cárie nos dentes dos filhos, $60,0 \%(n=12)$ deles apresentaram tal patologia, porém sem relevância estatística $(p=0,366)$.

Quando avaliada a escolaridade do membro da família que ensinou o filho sobre saúde bucal, 90,0\% $(n=9)$ dos que frequentaram o ensino médio completo foram os que instruíram mais ativamente. 0 estudo mostrou relevância estatística entre a escolaridade e a instrução de higiene bucal, demonstrada na Tabela $2,(p=0,043)$. 


\section{DISCUSSÃO}

O objetivo deste estudo foi avaliar o conhecimento dos pais ou responsáveis, sobre a saúde bucal dos seus filhos com necessidades especiais.

Um estudo transversal realizado com 23 crianças $^{12}$, no Centro Integrado de Educação Especial apontou como principal diagnóstico a paralisia cerebral (38,5\%), resultado semelhante da pesquisa com 43 cuidadores da Cidade de João Pessoa/PA, onde 36,0\% também a possuíam¹3. Divergindo do nosso estudo onde se observou uma maior prevalência de pacientes com Síndrome de Down (25,0\%). A paralisia cerebral atinge duas crianças para cada 1.000 nascidos vivos, sendo considerada a mais comum ${ }^{14,15}$.

Em nosso estudo, a mediana de idade dos PNE, encaminhados ao dentista pelo médico pediatra, foi de 1,00 (1,00-4,25) meses, corroborando o estudo sobre a percepção da saúde bucal dos bebês e conduta de 24 pediatras de Passo Fundo/RS, onde 54,2\% deles encaminham a criança aos 12 meses de vida ao cirurgião-dentista ${ }^{16}$.

Uma pesquisa na Paraíba, de abordagem indutiva ${ }^{13}$, constatou que $30,0 \%$ das crianças já foram ao dentista, sendo a primeira consulta por volta dos quatro anos (9,0\%). Os resultados de um estudo com 41 portadores de paralisia cerebral, de um a doze anos, das instituições de educação especial do município de Pelotas/RS, indicaram que a primeira consulta ao dentista ocorreu após os quatro anos de idade $(62,3 \%)^{17}$. No nosso estudo, a mediana encontrada referente à primeira visita do PNE ao dentista foi de cinco anos. Entretanto, preconiza-se que seja entre 6 a 12 meses essa primeira visita, fundamentada na facilidade de obter hábitos saudáveis, além de que, nesse estágio, a criança tem a erupção do primeiro dente decíduo, podendo, assim, prevenir possíveis patologias bucais ${ }^{18,19}$.

O tratamento odontológico do PNE requer alterações, seja na conduta clínica, como também na compreensão das dificuldades físico/motoras e de acesso aos serviços de saúde ${ }^{20}$. Quando os pais ou responsáveis foram questionados sobre o motivo que levou seu filho ao dentista pela primeira vez, $65,6 \%$ responderam "consulta de rotina".

Num estudo 7 transversal com 74 participantes, $45,6 \%$ dos PNE foram colaborativos durante otratamento odontológico e $21,1 \%$ choraram. Esses dados corroboram o nosso estudo, uma vez que muitos pacientes $(43,8 \%)$ apresentaram um comportamento calmo no momento da consulta e apenas $21,9 \%$ choraram. Os resultados apresentados fortalecem o conceito do atendimento precoce, pois, além de prevenir doenças bucais, proporciona maior conforto ao paciente no decorrer do atendimento.

Sobre o local de atendimento para o tratamento dentário, num estudo realizado com portadores de necessidades especiais, $73,7 \%$ procuraram as instituições públicas 7 . Semelhante ao nosso estudo, onde $43,8 \%$ responderam buscar atendimento odontológico na rede pública e consultório particular, porém divergente do estudo de Cardoso et al. ${ }^{13}$ (2011), com 43 cuidadores, onde o atendimento foi no setor privado (15\%). Outro estudo com 48 usuários da Associação Catarinense para Integração do Cego (Acic), em Florianópolis/SC (2007), relatou que 58,33\% utilizam o serviço público, enquanto $22,91 \%$ procuram atendimento particular 5 . Grande parte da população brasileira procura pelo atendimento público, em muitos casos sendo a única opção de tratamento. A Constituição Federal garante o direito de todos à saúde e a Convenção sobre os Direitos das Pessoas com Deficiência (2006) tem o intuito de certificar o acesso aos serviços de saúde em condições de igualdade ${ }^{21}$. 
CRESCÊNCIO MCC, CRISTIANO DP, SIMÕES PW. SONEGO FGF. Análise do Conhecimento de Pais ou Responsáveis sobre a Saúde Bucal dos

Filhos com Necessidades Especiais. Rev. Odontol. Univ. Cid. São Paulo 2018 abr/jun 30(2) 144-156

ISSN 1983-5183

O comprometimento motor do indivíduo causa dificuldades em determinadas atividades diárias, incluindo a higiene bucal'. Cerca de $78,1 \%$ dos pais relatam que seus filhos não possuem deficiência motora. Divergindo de um estudo em Araras (SP) que verificou que 59,5\% dos alunos da Escola Municipal de Ensino Especial Luiza Helena Remédio apresentavam falta de coordenação motora ${ }^{22}$.

O questionário aplicado com 100 pais de alunos matriculados na Associação de Pais e Amigos dos Excepcionais em 2011 relatou que 41,0\% deles são responsáveis pela escovação dental de seus filhos ${ }^{23}$. No nosso estudo o percentual da participação dos pais ou responsáveis na higiene bucal dos filhos foi de 51,6\%. Os familiares devem receber atenção especial, por meio de programas educativos, apresentando diferentes métodos que os auxiliem nesse cuidado ${ }^{24}$.

O método de escovação é simples e eficaz para remover a placa bacteriana da superfície dentária ${ }^{25}$. Num estudo envolvendo 100 pais no município de Ji-Paraná, RO, em 201123, 41,0\% deles informaram escovar os dentes do seu filho PNE três vezes ao dia. No nosso estudo, 71,9\% dos participantes escovam os dentes de seus filhos duas a três vezes ao dia. Na correlação do gênero dos participantes e a quantidade de escovação dental realizada, por dia, pelos pacientes, houve significância estatística $(p=0,007)$. Sabe-se do papel importante do cuidador, na manutenção da saúde em geral e bucal do PNE, devido às condições inerentes a sua doença que o impedem de realizar determinadas atividades ${ }^{12}$.

Segundo o Instituto Brasileiro de Geografia e Estatística (IBGE), dentre 10\% dos portadores de necessidades especiais somente $3 \%$ têm acesso ao atendimento odontológico, ou seja, cerca de 480 mil pacientes $^{12}$. Se compararmos com a situação de outros países, $13 \%$ a $75 \%$ das crianças especiais apresentam dificuldades. Esse fator pode estar relacionado à incapacidade dos profissionais em atender PNE ${ }^{13}$. No Condado Oakland Country, do estado de Michigan, um estudo com 117 estudantes apontou que 34,2\% têm dificuldade em manter a saúde bucal pela indisposição dos dentistas para tratar pacientes com deficiência, e com aumento da idade piora essa situação ${ }^{26}$. Na nossa pesquisa, a maior dificuldade para manter a saúde bucal do PNE seria encontrar um dentista que o atenda, assim como passar o fio dental 34,4\% ( $n=11$ ).

No contexto familiar, a figura materna possui maior influência quanto às mudanças no comportamento dos integrantes da família ${ }^{6,27}$. Em nosso estudo se observou predominância das mães quanto à instrução de seus filhos sobre saúde bucal (75\%). E ainda, foi obtida associação com significância estatística $(p=0,043)$ entre a escolaridade e responsabilidade dos pais pela saúde bucal dos filhos. Quanto maior a escolaridade, maior o acesso à informação vigente, e ao cuidado bucal. Com isso, os pais acabam participando ativamente da manutenção saúde oral de seus filhos. Um estudo em Ribeirão Preto/SP24 verificou que $85 \%$ das mães são responsáveis pela higiene oral do PNE. Fatores como a falta de instrução, de estímulo e de curiosidade dos familiares sobre a saúde bucal de seus filhos causam o agravamento das alterações bucais preexistentes ${ }^{12}$. A negligência da saúde bucal dos filhos, pelos pais ou responsáveis ocorre pelas dificuldades físicas, mentais e sociais, e pela priorização de outros cuidados ${ }^{28}$.

Uma pesquisa com 102 responsáveis ${ }^{29}$ classificou a saúde bucal dos filhos como boa (71,7\%), corroborando nosso estudo, onde metade dos pais a consideram boa e diferindo de um estudo realizado em 2011, onde somente $38 \%$ dos filhos têm saúde bucal satisfatória7. O governo federal, na tentativa de suprir as necessidades dos pacientes com determinadas limitações, lançou a Rede de Cuidados à Pessoa com Deficiência, tendo como objetivo promover assistência humanizada e o acesso facilitado ${ }^{30}$.

O nosso estudo apresentou dados quanto aos fatores que facilitariam a manutenção da saúde bucal do PNE; 38,7\% dos pais referem-se ao aumento de centros de referência habilitados e maior número de 
ISSN 1983-5183

profissionais, além do trabalho multidisciplinar (19,4\%). Já um estudo descritivo e transversal ${ }^{7}$ com 74 pais/ responsáveis relata a necessidade de maior número de profissionais e centros habilitados (12,3\%). Esses dados remetem à desinformação da comunidade sobre os serviços de média complexidade no setor público.

Com relação à renda mensal da família, um estudo ${ }^{31}$ em Natal/RN, 2010, demonstrou que 78,6\% têm renda inferior a um salário mínimo. Diferente da nossa pesquisa, onde $51,6 \%$ deles recebem até dois salários mínimos. Geralmente, os familiares costumam gastar além da renda mensal, para manutenção da saúde geral do PNE e, dessa forma, a assistência odontológica fica em segundo plano ${ }^{28}$.

Um estudo em Ponta Grossa/PR em 2007, com 40 crianças com síndrome de Down e seus pais, verificou que $85 \%$ apresentaram cárie 3 . Nesse estudo, a frequência quanto à doença cárie foi de $68,8 \%$. Sabe-se que muitos pacientes fazem uso frequente de medicamentos, sendo estes, muitas vezes, ricos em açúcares e que, associados à limitação de abertura de boca de alguns pacientes e à dificuldade de uma correta higiene bucal, propiciam o desenvolvimento dessa patologia ${ }^{32}$. Sobre a doença periodontal, em nosso estudo 75,0\% dos PNE não apresentaram a patologia, corroborando a percepção dos pais, pois $65,6 \%$ deles relatam que seus filhos não possuem sangramento gengival.

Os PNE possuem a saúde bucal precária, pela ineficiência na destreza manual, dificuldade em abrir a boca ou expelir o creme dental ${ }^{3}$. Dessa forma, um dos objetivos específicos da pesquisa era capacitar os pais e consequentemente seus filhos sobre os diversos métodos de higiene oral adaptados dentro das respectivas necessidades dos pacientes ${ }^{33}$. Muitos alegaram desconhecer os recursos e técnicas para efetuação da higiene bucal do PNE. Ao término da capacitação foi entregue uma cartilha, para reforçar a apreensão do conteúdo exposto.

\section{CONCLUSÃO}

Grande parte dos participantes eram mães que possuíam o ensino médio completo, moravam em casas próprias e que, ao responderem o questionário, apresentaram conhecimento satisfatório sobre a saúde bucal de seus filhos com necessidades especiais. Porém observou-se que mais da metade dos filhos apresentaram doença cárie instalada, mesmo eles tendo classificado a própria condição bucal como boa. As ações educativas, voltadas aos pais ou responsáveis, são de suma importância na diminuição do risco de agravos na cavidade bucal de seus filhos. 


\section{REFERÊNCIAS}

1. PEREIRA LM, Mardero E, Ferreira SH, Kramer PF, Cogo RB. Atenção odontológica em pacientes com deficiências: a experiência do curso de Odontologia da ULBRA (Canoas/RS). Stomatos 2010 jun.-dez.;16(31):92-9.

2. INSTITUTO Brasileiro de Geografia e Estatística - IBGE. Censo Demográfico 2010: características gerais da população, religião e pessoas com deficiência. 2012 [Acesso em: 05 jun. 2018]; Disponível em: https://biblioteca.ibge.gov.br/visualizacao/periodicos/94/ cd_2010_religiao_deficiencia.pdf.

3. TEITELBAUM AP, Sabbagh-Haddad A, Czlusniak GD, Pinto MHB, Santos FA. Ação de dentifrícios experimentais sobre a saúde bucal de crianças com síndrome de Down. IJD, Int $j$ dent 2010 9(3):128-35.

4. NASILOSKI KS, Silveira ERD, César Neto JB, Schardosim LR. Avaliação das condições periodontais e de higiene bucal em escolares com transtornos neuropsicomotores. Rev Odontol UNESP 2015 mar.-abr.;44(2):103-7.

5. CERICATO GO, Lamha APSF. Hábitos de saúde bucal de portadores de deficiência visual no contexto da saúde coletiva. RFO UPF 2012 ago.;17(2):137-44.

6. MARCELINO G, Parrilha VA. Educação em saúde bucal para mães de crianças especiais: um espaço para a prática dos profissionais de enfermagem. Cogitare Enfermagem 2007 12(1):37-43.

7. QUEIROZ FS, Rodrigues MMLF, Cordeiro Junior GA, Oliveira AB, Oliveira JD, Almeida ER. Avaliação das condições de saúde bucal de portadores de necessidades especiais. Rev Odontol UNESP 2014 nov.-dez.;43(6):396-401.

8. BRASIL. Ministério da Saúde. Programa Brasil Sorridente. 2004 [Acesso em: 06 jun. 2018]; Disponível em: http://dab.saude.gov.br/portaldab/ape brasil sorridente.php.

9. PAULETO ARC, Pereira MLT, Cyrino EG. Saúde bucal: uma revisão crítica sobre programações educativas para escolares. Ciênc saúde coletiva 2004 9(1):121-30.

10. MARRA PS. Dificuldades encontradas pelos responsáveis, para manter a saúde bucal em portadores de necessidades especiais [Dissertação]. Duque de Caxias, RJ: Universidade do Grande Rio "professor José de Souza Herdy"; 2007.

11. PEREIRA CB. Percepção sobre a educação em saúde bucal de crianças surdas nas escolas especiais de São Paulo [Dissertação]. São Paulo Universidade de São Paulo; 2010.

12. SOUSA IF, Ferreira DLA, Neto JMM. Sensibilização de pais de crianças com necessidades especiais. Rev Gestão \& Saúde 2014 5(4):16.

13. CARDOSO AMR, Brito DBA, Alves VF, Padilha WWN. O acesso ao cuidado em saúde bucal para crianças com deficiência motora: perspectivas dos cuidadores. Pesq Bras Odontoped Clin Integr 2011 11(4):593-99. 
ISSN 1983-5183

14. BRASIL. Ministério da Saúde. Secretaria de Atenção à Saúde. Diretrizes de atenção à pessoa com paralisia cerebral. Brasília: Ministério da Saúde; 2013 [Acesso em: 04 jun. 2018]; Disponível em: http://bvsms.saude.gov.br/bvs/publicacoes/diretrizes atencao paralisia cerebral.pdf.

15. O'SHEA TM. Diagnosis, treatment, and prevention of cerebral palsy. Clinical obstetrics and gynecology 2008 Dec;51(4):816-28.

16. NUNES OP, Brusco EH, Brusco LC, Perussolo B, Patussi EG. Percepções e condutas de médicos pediatras com relação à promoção. RGO - Rev Gaúcha Odontol, Porto Alegre 2011 abr./jun.;59(2):251-7.

17. GUERREIRO PO, Garcias GL. Diagnóstico das condições de saúde bucal em portadores de paralisia cerebral do município de Pelotas, Rio Grande do Sul, Brasil. Ciênc saúde coletiva 2009 14(5):1939-46.

18. KRAMER PF, Ardenghi TM, Ferreira S, Fischer LA, Cardoso L, Feldens CA. Utilização de serviços odontológicos por crianças de 0 a 5 anos de idade no Município de Canela, Rio Grande do Sul, Brasil. Cad Saúde Pública 2008 24(1):150-6.

19. FERNANDES DSC, Klein GV, Lippert AO, Medeiros NG, Oliveira RP. Motivo do atendimento odontológico na primeira infância. Stomatos 2010 16(30):04-10.

20. OLIVEIRA ALBM, Giro EMA. Importância da abordagem precoce no tratamento odontológico de pacientes portadores de necessidades especiais. Odonto 2011 jul./ dez.;19(38):45-51.

21. PISANESCHI E, Pedotti MAC. A política nacional de saúde para pessoas com deficiência: inclusão. Rev Educ esp 2010 5(2):54-63.

22. FLÓRIO FM, Basting RT, Salvatto MV, Migliato KL. Saúde bucal em indivíduos portadores de múltiplas deficiências. RGO, Porto Alegre 2007 jul./set.;55(3):251-6.

23. SOARES J, Volpato LER, Castro PHS, Lambert NA, Borges ÁH, Carvalhosa AA. Avaliação do conhecimento sobre saúde bucal de pais e cuidadores. J Health Sci Inst 2013 31(3):23943.

24. Ruviére DB, Queiroz AM, Serrano KVD, Freitas AC, Silva FWGP, Nelson-Filho P. Toothbrushing in patients with neurological and / or motor disorders. Odontol clín-cient 2010 abr.-jun. ;9(2):135-7.

25. CHIBINSKI CA, Grando K, Fanchin PT, Campagnoli E, Santos FA, Wambier DS. Descontaminação de escovas dentais utilizadas por crianças portadoras de necessidades especiais: análise microbiológica. RSBO 2011 8(1):145-52.

26. WILLIAMS JJ, Spangler CC, Yusaf NK. Barriers to dental care access for patients with special needs in an affluent metropolitan community. Special care in dentistry : official publication of the American Association of Hospital Dentists, the Academy of Dentistry for the Handicapped, and the American Society for Geriatric Dentistry 2015 Jul-Aug;35(4):190-6. 
ISSN 1983-5183

27. SANTOS BMO, Aquino DJN, Pires-De-Souza FCP, Almeida GL, Garcia LFR. Assistência odontológica a portadores de necessidades especiais sob a ótica dos cuidadores. Cienc Odontol Bras 2009 abr./jun.;12(2):49-56.

28. MARTÍNEZ Menchaca HR, Treviño Alanís G, Rivera Silva G. Guía para el cuidado de la salud oral en pacientes con necesidad de cuidados especiales de salud en México. Rev ADM 2011 68(5):222-8.

29. MARRA OS, Miasato JM. A saúde bucal do paciente especial e sua relação com o nível sócio-econômico dos pais. Rev bras odontol 2008 65(1):27-30.

30. CHAVES SCL, Barros SG, Cruz DN, Figueiredo ACL, Moura BLA, Cangussu MCT. Política nacional de saúde bucal: fatores associados à integralidade do cuidado. Rev Saúde Pública 2010 44(6):1005-13.

31. BARROS GM, Santos MTBR. Socioeconomic profile of patients treated at the children's rehabilitation center of Natal, state of Rio Grande do Norte, Brazil. Pesqui bras odontopediatria clín integr 2014 14(4):303-11.

32. NEVES BG, Pierro VSS, Maia LC. Percepções e atitudes de responsáveis por crianças frente ao uso de medicamentos infantis e sua relação com cárie e erosão dentária. Ciênc saúde coletiva 2007 out.;12(5):1295-300.

33. HARTWIG AD, Silva Junior IF, Stüermer VM, Schardosim LR, Azevedo MS. Recursos e técnicas para a higiene bucal de pacientes com necessidades especiais. Rev Virtual ACBO 2015 4(3):1-10.

RECEBIDO EM 29/09/2017

ACEITO EM 04/04/2018 